\title{
Ordered Arrays of Size-Selected Oxide Nanoparticles
}

\author{
Luca Gragnaniello, ${ }^{1,3}$ Teng Ma, ${ }^{1}$ Giovanni Barcaro, ${ }^{2}$ Luca Sementa, ${ }^{2}$ Fabio R. Negreiros, ${ }^{2}$ Alessandro Fortunelli, ${ }^{2}$ \\ Svetlozar Surnev, ${ }^{1}$ and Falko P. Netzer ${ }^{1}$ \\ ${ }^{1}$ Surface and Interface Physics, Institute of Physics, Karl-Franzens University Graz A-8010 Graz, Austria \\ ${ }^{2}$ CNR-IPCF, Istituto per i Processi Chimico-Fisici del CNR, I-56124 Pisa, Italy \\ ${ }^{3}$ École Polytechnique Fédérale de Lausanne (EPFL), Lausanne CH-1015 Switzerland
}

(Received 5 February 2012; published 11 May 2012)

\begin{abstract}
A bottom-up approach to produce a long-range ordered superlattice of monodisperse and isomorphic metal-oxide nanoparticles (NP) supported onto an oxide substrate is demonstrated. The synthetic strategy consists of self-assembling metallic NP on an ultrathin nanopatterned aluminum oxide template followed by a morphology-conserving oxidation process, and is exemplified in the case of $\mathrm{Ni}$, but is generally applicable to a wide range of metallic systems. Both fully oxidized and core-shell metal-metal-oxide particles are synthesized, up to 3-4 $\mathrm{nm}$ in diameter, and characterized via spectroscopic and theoretical tools. This opens up a new avenue for probing unit and ensemble effects on the properties of oxide materials in the nanoscale regime.
\end{abstract}

DOI: 10.1103/PhysRevLett.108.195507

PACS numbers: 81.16.Pr, 61.46.Df, 75.75.Cd, 81.16.Rf

Metal-oxide nanoparticles (NP) find widespread interest in fundamental science and bear promise for high-level applications in many emerging fields of the advanced nanotechnologies, from nanospintronics and high-density magnetic storage devices [1-3] to chemical sensing [4,5] and nanocatalysis [6,7]. The size, shape, and mutual interactions among the particles play a key role in determining the novel properties of these nanosized oxide materials $[8,9]$. For example, oxide NP may display anomalous magnetic behavior such as ferromagnetism vs antiferromagnetism in the bulk [2,3] or size-dependent magnetic structures and unusual spin couplings $[8,10]$. Model systems of NP with atomic-level control of size, shape, chemical composition, and interparticle distance are therefore crucial to clarify the emergent phenomena in oxide materials at the nanometer scale, and to disentangle the effects of the various intra-, inter- and support-particle interactions. However, such systems are difficult to prepare especially for particles below 50-100 nm, which are not accessible to lithographic techniques [5], while the traditional wet chemical methods only allow moderate design variations [11]. Here we describe the fabrication of one such system: an ordered superlattice of monodisperse and isomorphic nickel oxide NP, and its characterization via spectroscopic and theoretical tools. Both fully oxidized and core-shell metal-metal-oxide particles (the latter of interest, e.g., for exchange bias effects [1]) are synthesized via a bottom-up template-directed self-assembly strategy, opening up a new avenue for probing unit and ensemble effects on the physical and chemical properties of oxide materials, from molecule-type cluster sizes to the solid state limit. Our strategy consists of self-assembling metallic NP on an ultrathin nanopatterned aluminum oxide template followed by a morphology-conserving oxidation process, thus producing a superlattice of oxide NP decoupled from a conducting metal substrate by an ultrathin oxide layer, with a very narrow size distribution and identical shapes. We illustrate the procedure for the case of $\mathrm{NiO} \mathrm{NP}$, but the method is general (it has been tested for $\mathrm{CoO}$ particles with equal success).

The ultrathin $\mathrm{Al}$ oxide layer, which is created by thermal oxidation on a $\mathrm{Ni}_{3} \mathrm{Al}(111)$ alloy single crystal surface, constitutes a well-ordered nanostructured template with holes that provide anchoring centers for the directed assembly of metal NP in a physical vapour deposition (PVD) process $[12,13]$. The structure of the $0.5 \mathrm{~nm}$ thick alumina $\mathrm{AlO}_{x}$ film on $\mathrm{Ni}_{3} \mathrm{Al}(111)$ contains holes at the corners of the $(\sqrt{67} \times \sqrt{67}) R 12.2^{\circ}$ unit cell reaching down to the metal substrate [14], in which up to three Pd atoms can be trapped; the latter can act as heterogeneous nucleation centers for Ni adatoms deposited subsequently by PVD, thereby creating a well-ordered hexagonal array of metal clusters [15] (the "seeding" of the alumina surface by predeposited Pd atoms is necessary to provide nucleation centers for the subsequent self-assembly of metal clusters [16]). The presence of a regular array of nucleation centers strongly reduces the size dispersion of epitaxially grown metal particles [17].

Figures 1(a) and 1(b) show scanning tunneling microscopy (STM) images of Ni NP on Pd-seeded $\mathrm{AlO}_{x} / \mathrm{Ni}_{3} \mathrm{Al}(111)$, prepared by deposition of $2.07 \times 10^{13} \mathrm{Pd}$ atoms $/ \mathrm{cm}^{2}$ at room temperature (corresponding to three $\mathrm{Pd}$ atoms per corner hole on average) followed by deposition of $\mathrm{Ni}$ atoms at $200^{\circ} \mathrm{C}$ (see Supplemental Material for further experimental details [18]). At a $\mathrm{Ni}$ coverage of $3.2 \times 10^{14}$ atoms $/ \mathrm{cm}^{2}$ [Fig. 1(a)] the surface is covered by Ni NP, with a particle density of approximately $3 \times 10^{12}$ particles $/ \mathrm{cm}^{2}$. The high-resolution image of the inset of Fig. 1(a) displays the so-called "network" structure of the alumina substrate 

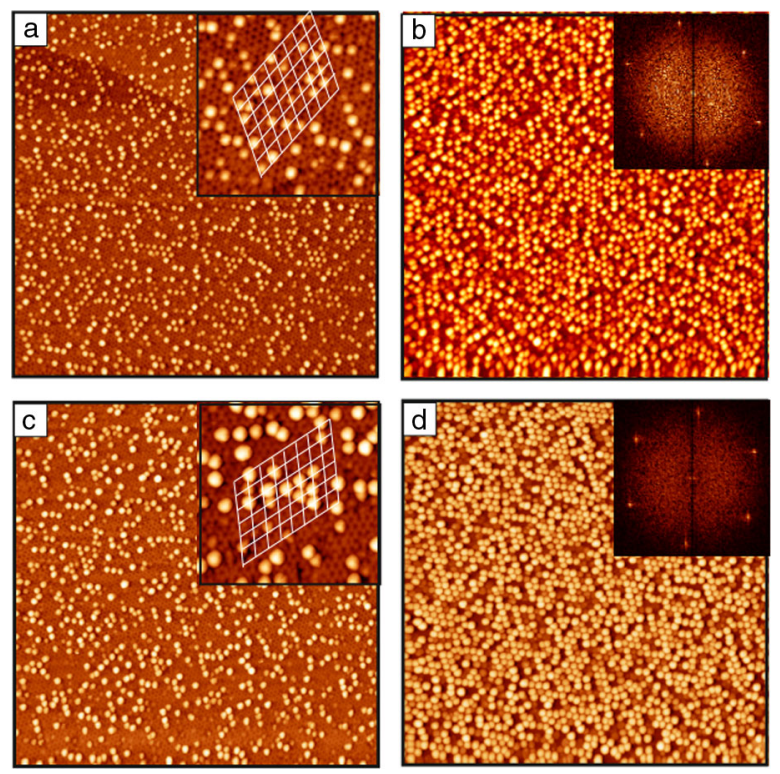

FIG. 1 (color online). STM images of Ni NP on Pd-seeded $\mathrm{AlO}_{x} / \mathrm{Ni}_{3} \mathrm{Al}(111)$, formed after deposition of $3.2 \times$ $10^{14} \mathrm{Ni}$ atoms $/ \mathrm{cm}^{2}$ (a) and $1.37 \times 10^{15} \mathrm{Ni}$ atoms $/ \mathrm{cm}^{2}$ (b) at $200{ }^{\circ} \mathrm{C}(200 \mathrm{~nm} \times 200 \mathrm{~nm}$; sample bias: $3.0 \mathrm{~V}$; tunneling current: $0.1 \mathrm{nA})$. STM images (c) and (d) were recorded on surfaces (a) and (b), respectively, after oxidation at $200{ }^{\circ} \mathrm{C}\left(p_{\mathrm{O}_{2}}=1 \times\right.$ $10^{-6}$ mbar, $<200 \mathrm{~L}$ ) producing $\mathrm{NiO}$ particles (see text). Insets: (a) and (c) high-resolution STM images $(50 \mathrm{~nm} \times 50 \mathrm{~nm} ; 3.0 \mathrm{~V}$; $0.1 \mathrm{nA}$ ); the crossing points of the white grid mark the corner holes of the $\mathrm{AlO}_{x}$ unit cell. (b) and (d) fast Fourier transforms (FFT) of (b) and (d), respectively.

[12] in between the Ni NP, with the lattice of the corner holes (the so-called "dot" structure [12]) indicated by the superimposed white grid. The majority of the Ni NP nucleates at Pd-seeded corner holes (the crossing points of the white grid), and exhibit average apparent heights of $0.83 \pm 0.17 \mathrm{~nm}$ and diameters of $1.9 \pm 0.7 \mathrm{~nm}$ as measured from STM (see below), suggesting roughly hemispherical shapes. At higher coverage $\left(1.37 \times 10^{15} \mathrm{Ni}\right.$ atoms $\left./ \mathrm{cm}^{2}\right)$, a highly ordered array of $\mathrm{Ni}$ NP is formed [Fig. 1(b)]: the fast Fourier transform (FFT) of the STM image (inset) reveals a periodicity of $4.1 \mathrm{~nm}$, identical to the dot lattice of the alumina template. The size of the particles has increased to $1.15 \pm 0.15 \mathrm{~nm}$ (height) and $2.6 \pm 0.3 \mathrm{~nm}$ (diameter), with a NP density of about $4.4 \times 10^{12}$ particles $/ \mathrm{cm}^{2}$, corresponding to a coverage of $\sim 60 \%$ of the corner holes by NP.

The transformation of metallic into oxidic NP requires a morphology-conserving oxidation procedure in order to maintain the superlattice character of the system. Exposing the metallic Ni NP to $\mathrm{O}_{2}\left(p_{\mathrm{O}_{2}}=10^{-6} \mathrm{mbar}\right)$ at $200^{\circ} \mathrm{C}\left(>200 \mathrm{~L}, 1\right.$ Langmuir $(\mathrm{L})=10^{-6}$ torr $\left.\cdot \mathrm{sec}\right)$ leads to the complete oxidation of the NP, as discussed below. Visual inspection of the STM images of Figs. 1(c) and 1(d), recorded after oxidation, reveals that the morphology (and the particle density) of the superlattice is maintained: the inset of Fig. 1(c) confirms that the oxidized Ni NP still occupy the corner hole sites, and the FFT of the inset of Fig. 1(d) gives evidence of the hexagonal dot lattice order of $4.1 \mathrm{~nm}$ periodicity.

The comparison of NP size before and after oxidation gives an indication of the chemical composition of the oxidation product [19]. NP diameter and height distribution plots before and after oxidation are displayed in Fig. 2(a). Two aspects are worth pointing out: first, the narrow distributions are only slightly wider after oxidation, confirming the morphology-conserving process; second, the size of the particles increases upon oxidation: the resulting ratio volume $_{\text {oxideNP }} /$ volume $_{\text {metalNP }}=1.57 \mathrm{com}$ pares favorably with the corresponding ratio volume $\mathrm{NiO}_{\mathrm{N}} /$ volume $_{\mathrm{Ni}}=1.66$ for rock-salt $\mathrm{NiO}$ and fcc Ni metal, suggesting that the Ni NP have been fully oxidized (additional data in the Supplemental Material, Fig. S1, [18]). The range of particle sizes which can be prepared by the present
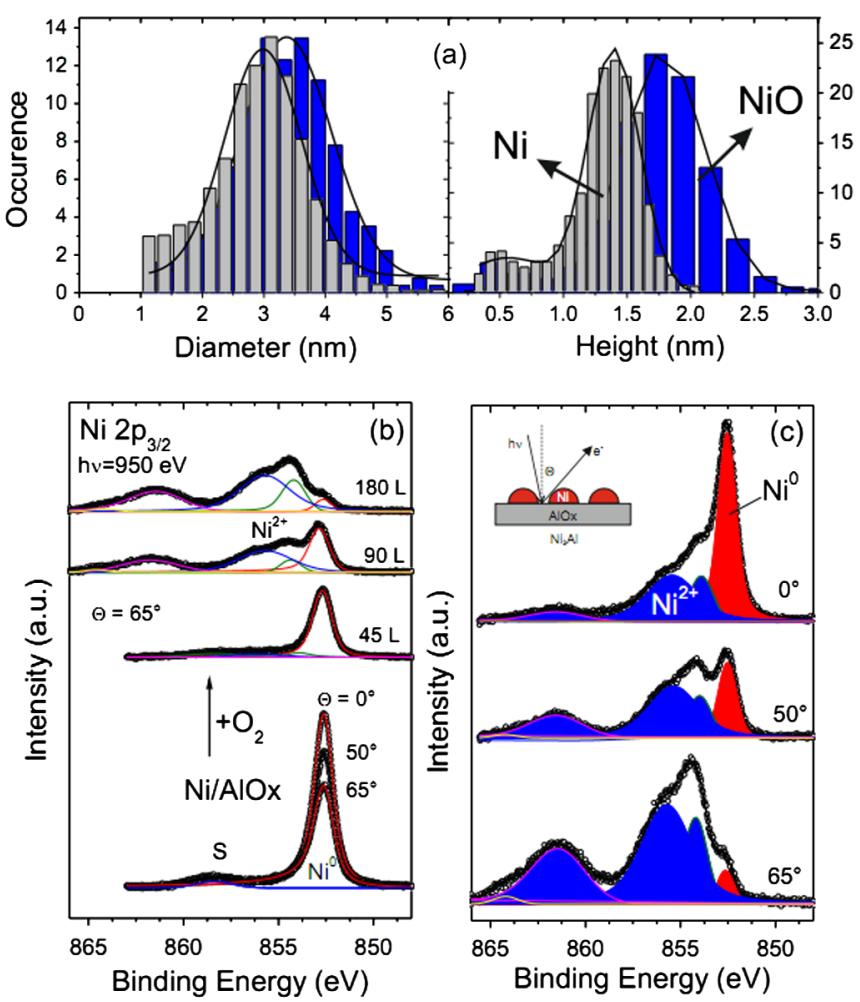

FIG. 2 (color online). (a) Diameter and height distribution of $\mathrm{Ni} \mathrm{NP}\left(\mathrm{Ni}\right.$ coverage: $1.37 \times 10^{15}$ atoms $/ \mathrm{cm}^{2}$ ) before (gray) and after (blue) oxidation, as determined from STM images of Figs. 1(b) and 1(d). (b) Ni $2 p_{3 / 2}$ core-level photoemission spectra taken at a grazing emission angle $\theta=65^{\circ}$ as a function of $\mathrm{O}_{2}$ exposure (in L) at $200^{\circ} \mathrm{C}$. The bottom set of spectra has been taken at various electron emission angles $\theta$. (c) $\mathrm{Ni} 2 p_{3 / 2}$ core-level spectra of the fully oxidized $\mathrm{Ni} \mathrm{NP}$ after $\mathrm{O}_{2}$ exposure of $180 \mathrm{~L}$ at various emission angles $\theta$. The spectral components due to metallic $\left(\mathrm{Ni}^{0}\right)$ and oxidic $\left(\mathrm{Ni}^{2+}\right)$ species are colored red and blue, respectively, (a sketch of the measuring geometry in the inset). 
method is restricted by the coalescence limit of the NP at a diameter of $\sim 4.1 \mathrm{~nm}$, the distance of corner hole nucleation centers in the dot structure of the $\mathrm{AlO}_{x}$ substrate, corresponding to $\approx 1600$ atoms per hemispherical particle.

The oxidation of the Ni NP has been followed as a function of $\mathrm{O}_{2}$ exposure by high-resolution emission-angle dependent Ni $2 p$ photoelectron spectroscopy, to vary the surface sensitivity of the method and to disentangle the $\mathrm{Ni}$ signal of the $\mathrm{NP}$ and that of the $\mathrm{Ni}_{3} \mathrm{Al}$ substrate. Figure 2(b) shows Ni $2 p_{3 / 2}$ core-level spectra of the pristine Ni NP system and after exposure to $\mathrm{O}_{2}$ at $200{ }^{\circ} \mathrm{C}$. The bottom spectra display the Ni $2 p_{3 / 2}$ emission of the pristine system as a function of the emission angle-see inset of Fig. 2(c) for a sketch of the experimental geometry. For $\theta=0^{\circ}$, the $\mathrm{Ni} 2 p$ signal is composed of the emission from both the $\mathrm{Ni}$ $\mathrm{NP}$ and the $\mathrm{Ni}_{3} \mathrm{Al}$ substrate due to the large escape depth of the photoelectrons, whereas at $65^{\circ}$ emission from the $\mathrm{Ni}$ NP prevails due to the high surface sensitivity at this geometry. After exposure to $45 \mathrm{~L} \mathrm{O}_{2}$, the $\mathrm{Ni} 2 p$ emission is still characteristic of metallic Ni NP, but after an $\mathrm{O}_{2}$ dose of $90 \mathrm{~L}$ the metallic emission has decreased and broad emission peaks at higher binding energy signal the presence of $\mathrm{Ni}^{2+}$ species. After $180 \mathrm{~L} \mathrm{O}_{2}$ exposure, the latter features are dominant and have reached saturation intensity whereas only a small $\mathrm{Ni}^{0}$ signal remains. The metallic character of $\mathrm{Ni}$ after $45 \mathrm{~L} \mathrm{O}_{2}$ exposure indicates a delay in the onset of NP oxidation and suggests that the oxidation process occurs in two steps, involving both the Ni NP and the $\mathrm{AlO}_{x} / \mathrm{Ni}_{3} \mathrm{Al}$ substrate as discussed below.

Figure 2(c) displays Ni 2 $p_{3 / 2}$ spectra of the oxidized Ni NP surface (180 $\mathrm{L} \mathrm{O}_{2}$ dose) as a function of the electron emission-angle $\theta$ and a decomposition analysis of the spectral profiles into individual components [18]. The $\mathrm{Ni}^{0}$ component at $852.5 \mathrm{eV}$ is pronounced at $\theta=0^{\circ}$ due to the large contribution from the substrate, but remains only as a weak shoulder in the $\theta=65^{\circ}$ spectrum, where the emission of the oxidized Ni NP in the binding energy range $854-865 \mathrm{eV}$ (denoted as $\mathrm{Ni}^{2+}$ ) is dominant. The spectral profile of the $\mathrm{Ni}^{2+}$ emission is complex with three major components, which agree well with published $\mathrm{NiO}$ spectra $[20,21]$. The $\mathrm{Ni}^{2+} 2 p$ emission profile confirms unambiguously the chemical nature of the $\mathrm{NP}$ as $\mathrm{NiO}$.

The oxidation process has been also quantified by comparing the angle dependent $\mathrm{Ni} 2 p$ emission intensities with model calculations. Figures 3(a) and 3(b) display integrated intensities of $\mathrm{Ni}^{0}$ and $\mathrm{Ni}^{2+}$ components, respectively, with $\mathrm{O}_{2}$ exposure at $200^{\circ} \mathrm{C}$ for different emission angles. For $\mathrm{O}_{2}$ exposure $<50 \mathrm{~L}$ no significant oxidation of the Ni NP occurs, as evident from the small increase of the $\mathrm{Ni}^{2+}$ intensities; the $\mathrm{Ni}^{0}$ intensities at all emission angles however become significantly suppressed, as a result of the thickening of the alumina film and the resulting attenuation of the signal from the substrate. With increasing $\mathrm{O}_{2}$ exposure, the $\mathrm{Ni}^{2+}$ intensity increases fast, with the most pronounced growth at the most surface-sensitive emission
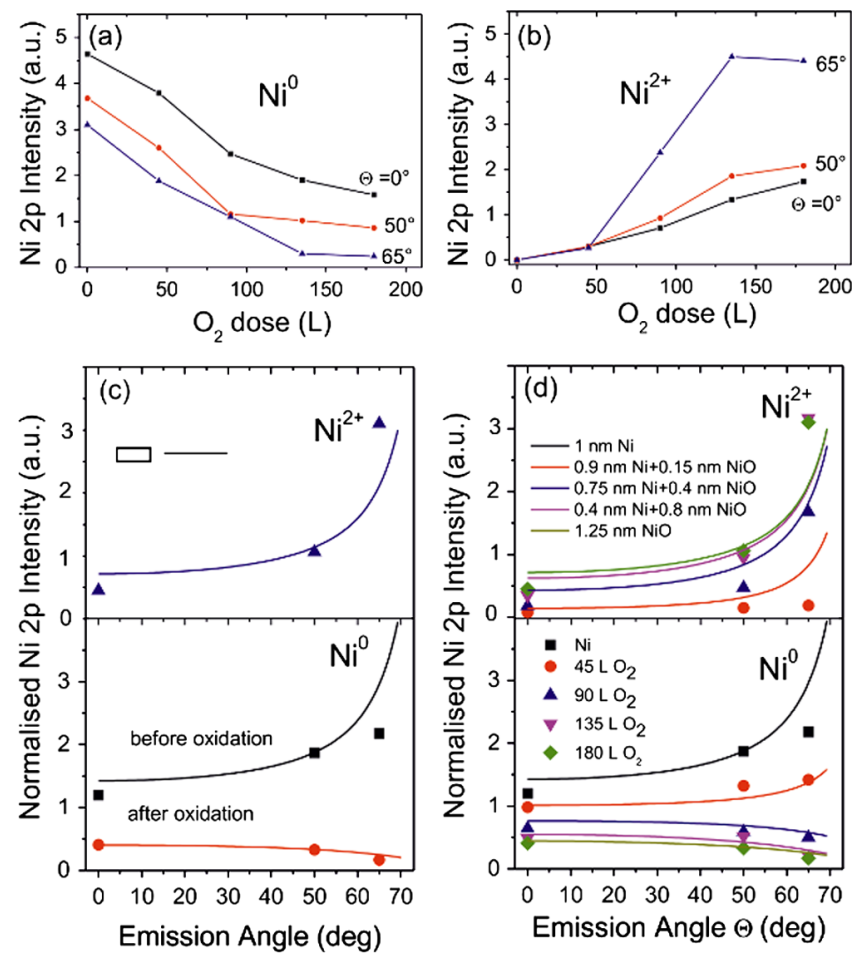

FIG. 3 (color online). Plots of the integrated Ni $2 p_{3 / 2}$ intensity at various emission angles versus the $\mathrm{O}_{2}$ dose (in $\mathrm{L}$ ) for the $\mathrm{Ni}^{0}$ (a) and $\mathrm{Ni}^{2+}$ (b) core-level components (Ni surface coverage: $1.37 \times 10^{15}$ atoms $/ \mathrm{cm}^{2}$ ). (c) Angular dependence of the integrated Ni $2 p_{3 / 2}$ intensity normalized to the respective values of the bare $\mathrm{AlO}_{x} / \mathrm{Ni}_{3} \mathrm{Al}(111)$ surface. The squares denote the normalized $\mathrm{Ni}^{0}$ intensity before oxidation, whereas the circles and triangles correspond to the $\mathrm{Ni}^{0}$ and $\mathrm{Ni}^{2+}$ intensities, respectively, after oxidation with $180 \mathrm{~L} \mathrm{O}_{2}$ at $200{ }^{\circ} \mathrm{C}$ ( $\mathrm{Ni}$ surface coverage as in (a),(b). The solid lines are curves calculated for particles with rectangular shapes (see Supplemental Material [18]). (d) Angular dependence of the normalized $\mathrm{Ni}^{0}$ (bottom panel) and $\mathrm{Ni}^{2+}$ (top panel) intensities for different $\mathrm{O}_{2}$ exposures. The solid curves are calculated for rectangular-box particles and increasing thickness of the NiO layer at the surface of the Ni particles.

geometry $\left(\theta=65^{\circ}\right)$, suggesting that the oxidation of the $\mathrm{Ni}$ NP starts at their surface. After an $\mathrm{O}_{2}$ dose of $180 \mathrm{~L}$, both the $\mathrm{Ni}^{0}$ and $\mathrm{Ni}^{2+}$ emission intensities reach saturation, indicating that the Ni NP are fully oxidized.

That oxidation proceeds from the surface into the core of the particles is confirmed by model calculations of angulardependent emission intensities (see Supplemental Material [18]). In Fig. 3(c) the normalized $\mathrm{Ni}^{0}$ and $\mathrm{Ni}^{2+}$ intensities measured prior and after oxidation with $180 \mathrm{~L} \mathrm{O}_{2}$ are plotted together with the intensities calculated for a surface coverage of $80 \%$ of rectangular-box $\mathrm{Ni}$ and $\mathrm{NiO} \mathrm{NP}$ with diameters of $3.0 \mathrm{~nm}$ and $3.5 \mathrm{~nm}$, respectively, as a function of the emission angle. The model reproduces well the angular dependence of the experimental $\mathrm{Ni}^{0}$ and $\mathrm{Ni}^{2+}$ intensities. This model was also used to calculate the angular-dependent $\mathrm{Ni}^{0}$ and $\mathrm{Ni}^{2+}$ intensities for increasing 
thickness of the oxide layer and compare it to the experimental data for different $\mathrm{O}_{2}$ exposures-see Fig. 3(d). The good agreement between the calculated and experiment data proves that the oxidation of the Ni NP proceeds in a layer-type fashion beginning at the NP surface, so that an oxide-shell/metal-core geometry is obtained for intermediate $\mathrm{O}_{2}$ doses.

The Al $2 p$ core-level spectra (Fig. 4) support the previous analysis and reveal further details of the oxidation process. Figure 4(a) shows Al $2 p$ core-level spectra of the bare substrate (bottom curve), after deposition of Ni NP (second level), and after the exposure to various $\mathrm{L}$ of $\mathrm{O}_{2}$ at $200{ }^{\circ} \mathrm{C}$ (higher levels). The $\mathrm{Al} 2 p$ spectrum of the $\mathrm{AlO}_{x} / \mathrm{Ni}_{3} \mathrm{Al}$ surface contains contributions from the $\mathrm{Ni}_{3} \mathrm{Al}$ bulk (labeled $B$ ), the $\mathrm{Ni}_{3} \mathrm{Al}-\mathrm{AlO}_{x}$ interface (labeled $\mathrm{I}$ ), and from the $\mathrm{AlO}_{x}$ top $\left(\mathrm{Al}_{\mathrm{S}}\right)$ and second layer $\left(\mathrm{Al}_{\mathrm{I}}\right)$ [22,23]. After Ni deposition, the $\mathrm{Al} 2 p$ signal decreases due to the $\mathrm{Ni}$ overlayer, but after $\mathrm{O}_{2}$ exposure the $\mathrm{AlO}_{x}$ components increase again significantly and shift to lower binding energy. This indicates that the $\mathrm{AlO}_{x} / \mathrm{Ni}_{3} \mathrm{Al}$ substrate also plays a crucial role in the overall reaction,
Core level shift (eV)

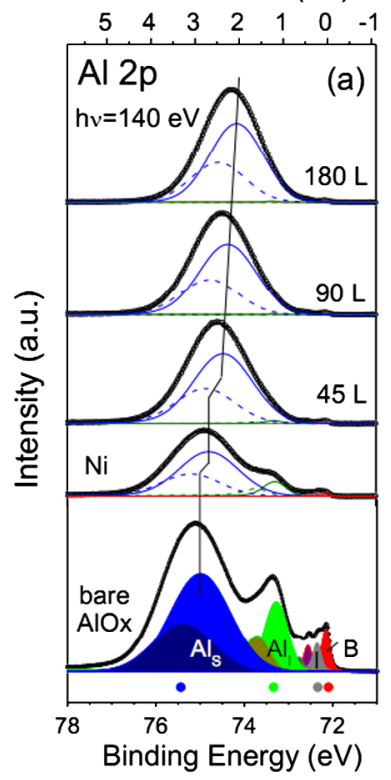

(b)

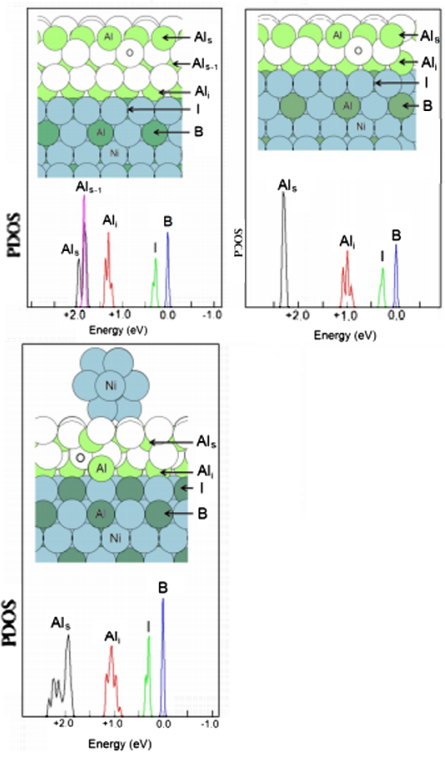

FIG. 4 (color online). (a) $\mathrm{Al} 2 p$ core-level photoemission spectra of the bare $\mathrm{AlO}_{x} / \mathrm{Ni}_{3} \mathrm{Al}$ surface (bottom), of the Ni NP covered surface ( $\mathrm{Ni}$ coverage: $1.37 \times 10^{15}$ atoms $/ \mathrm{cm}^{2}$ ), and after various $\mathrm{O}_{2}$ exposures. The different core-level components together with their corresponding theoretical binding energy positions predicted by using a final-state excited pseudopotential approach (filled circles at the bottom) are described in the text. (b) Structural models and PDOS for model systems: bare $A B C A$ bilayer alumina substrate (right side), same substrate with a $\mathrm{Ni}_{13}$ particle deposited on top (bottom), alumina substrate oxidized and thickened to an $A B C A B C$ trilayer (top left side). The various types of $\mathrm{Al}$ atoms are highlighted in the structural models, and the corresponding peaks in the PDOS around the Al $2 p$ level are also reported. in agreement with the delayed onset of the oxidation of the Ni NP in Fig. 2.

To validate the spectroscopic assignments and for a deeper understanding of the oxidation process, density functional (DF) calculations [24] were performed (see Supplemental Material [18] for the computational details). In a simplified model, shown on the right side of Fig. 4(b), the oxide slab is composed of four layers alternating $\mathrm{Al}$ and $\mathrm{O}$ atoms in the order $\mathrm{Al}_{i}-\mathrm{O}_{i}-\mathrm{Al}_{s}-\mathrm{O}_{s}$ starting from the $\mathrm{Ni}_{3} \mathrm{Al}$ alloy surface. The $\mathrm{Al}_{i}$ interfacial layer has 2 atoms per $\mathrm{Ni}_{3} \mathrm{Al}(111)$ unit cell arranged in a honeycomb lattice. The remaining layers all have 3 atoms per unit cell forming fcc (111) planes succeeding according to an $A B C A$ stacking (an $A B B A$ stacking was also considered producing qualitatively similar results, see Supplemental Material [18]). The total stoichiometry of the oxide is $\mathrm{Al}_{5} \mathrm{O}_{6}$ and is close to the real one [14], while the structure of these models resembles that of pseudoepitaxial regions in the $\mathrm{AlO}_{x} / \mathrm{Ni}_{3} \mathrm{Al}(111)$ complete cell. On the right side of Fig. 4(b) the projected density of states (PDOS) around the $\mathrm{Al} 2 p$ components is also displayed, showing a smooth progression of the Al $2 p$ core-levels to higher binding energies as one moves away from the bulk alloy, in fair agreement with experiment. The results of more accurate calculations using a final-state excited pseudopotential approach [25] are shown as filled circles at the bottom of Fig. 4(a): the agreement with experiment is excellent. The attenuation of the Al spectra and the shift to lower binding energies by roughly $0.3 \mathrm{eV}$ upon deposition of $\mathrm{Ni} \mathrm{NP}$ [Fig. 4(a)] is in tune with the PDOS of a model system composed of a $\mathrm{Ni}_{13}$ particle deposited on the $A B C A$ alumina substrate shown at the bottom of Fig. 4(b): the $\mathrm{Al}_{s}$ peak fragments and generates a component roughly $0.2-0.3 \mathrm{eV}$ lower in energy (corresponding to $\mathrm{Al}_{s}$ atoms underneath the $\mathrm{Ni}_{13}$ particle). This is due to a charge transfer from the substrate to the Ni NP causing the shift of the Al core levels to lower binding energies. The further shift occurring at $45 \mathrm{~L} \mathrm{O}_{2}$-before appreciable oxidation of the Ni NP_can be explained as due to a thickening of the oxide layer. A structural model for this phenomenon is shown on the top left side of Fig. 4(b), and consists of an $A B C A B C$ oxide slab obtained by adding an $\mathrm{Al}$ and a O layer in fcc stacking to the previous $A B C A$ model. From the PDOS also reported on the top left side of Fig. 4(b) we observe a shift to lower binding energies, a merging of the $\mathrm{Al}_{s-1}$ and $\mathrm{Al}_{s}$ peaks and an approaching of the $\mathrm{Al}_{i}$ peak to the Al-surface ones, in fair agreement with experiment. The thickening of the $\mathrm{AlO}_{x}$ layer in the presence of Ni NP obviously requires the dissociation of $\mathrm{O}_{2}$ on the NP followed by spillover to the oxide surface and penetration through the alumina to the $\mathrm{Ni}_{3} \mathrm{Al}$ interface, where an additional Al-O layer is generated [26].

In conclusion, we have shown that following a bottomup strategy based on a morphology-conserving oxidation of template-directed assemblies of metal aggregates it is 
possible to prepare ordered arrays of size-selected and isomorphic NP up to 3-4 $\mathrm{nm}$ in diameter either fully oxidized or in a metal-core/oxide-shell configuration, as proven by spectroscopic and theoretical characterization. The method is exemplified in the case of Ni NP, but is generally applicable to a wide range of metallic systems. The oxide nano-objects so produced may be regarded as nanolaboratories, in which size, shape, composition, particle-substrate, and particle-particle interaction effects on the properties of oxide materials can be investigated in the extreme nanoscale régime.

This work has been supported by the ERC Advanced Grant SEPON. Francesco Allegretti, Technical University Munich, is gratefully acknowledged for discussions concerning the XPS analysis. Calculations were performed at CINECA within the ISCRA OAMONUA project.

[1] V. Skumryev, S. Stoyanov, Y. Zhang, G. Hadjipanayis, D. Givord, and J. Nogués, Nature (London) 423, 850 (2003).

[2] S. K. Nayak and P. Jena, J. Am. Chem. Soc. 121, 644 (1999).

[3] G. H. Lee, S. H. Huh, J. W. Jeong, B. J. Choi, S. H. Kim, and H.-C. Ri, J. Am. Chem. Soc. 124, 12094 (2002).

[4] A. Kolmakov and M. Moskovits, Annu. Rev. Mater. Res. 34, 151 (2004).

[5] Y. Lei, W. K. Chim, J. Weissmüller, G. Wilde, H. P. Sun, and X. Q. Pan, Nanotechnology 16, 1892 (2005).

[6] J. A. Rodriguez, S. Ma, P. Liu, J. Hrbek, J. Evans, and M. Pérez, Science 318, 1757 (2007).

[7] P. Li, D. E. Miser, S. Rabiei, R. T. Yadav, M. R. Hajaligol, Appl. Catal. 43, 151 (2003).

[8] R. H. Kodama, S. A. Makhlouf, and A. E. Berkowitz, Phys. Rev. Lett. 79, 1393 (1997).

[9] J. Cheon, N.-J. Kang, S.-M. Lee, J.-H. Lee, J.-H. Yoon, and S.J. Oh, J. Am. Chem. Soc. 126, 1950 (2004).
[10] S. Morup, D. E. Madsen, C. Frandsen, C. R. H. Bahl, and M. F. Hansen, J. Phys. Condens. Matter 19, 213202 (2007).

[11] Y. Xia, B. Gates, Y. Yin, and Y. Lu, Adv. Mater. 12, 693 (2000).

[12] S. Degen, C. Becker and K. Wandelt, Faraday Discuss. 125, 343 (2004).

[13] L. Gavioli, E. Cavaliere, S. Agnoli, G. Barcaro, A. Fortunelli, G. Granozzi, Prog. Surf. Sci. 86, 59 (2011).

[14] M. Schmid, G. Kresse, A. Buchsbaum, E. Napetschnig, S. Gritschneder, M. Reichling, and P. Varga, Phys. Rev. Lett. 99, 196104 (2007).

[15] A. Buchsbaum, M. De Santis, H. C. N. Tolentino, M. Schmid, and P. Varga, Phys. Rev. B 81, 115420 (2010).

[16] G. Hamm, C. Becker and C. R. Henry, Nanotechnology 17, 1943 (2006).

[17] C. Lee and A. L. Barabasi, Appl. Phys. Lett. 73, 2651 (1998).

[18] See Supplemental Material at http://link.aps.org/ supplemental/10.1103/PhysRevLett.108.195507 for the decomposition analysis of the Ni $2 p$ spectra.

[19] Issues connected with the tunneling voltage dependent apparent particle height and tip convolution effects [12] were corrected by adopting the procedure outlined in Ref. [15].

[20] M. A. van Veenendaal and G. A. Sawatzky, Phys. Rev. Lett. 70, 2459 (1993).

[21] I. Preda, A. Gutiérrez, M. Abbate, F. Yubero, J. Méndez, L. Alvarez, L. Soriano, Phys. Rev. B 77, 075411 (2008).

[22] E. Vesselli, A. Baraldi, S. Lizzit, and G. Comelli, Phys. Rev. Lett. 105, 046102 (2010).

[23] N. M. Martin et al., Phys. Rev. B 83, 125417 (2011).

[24] P. Giannozzi et al., J. Phys. Condens. Matter 21, 395502 (2009).

[25] L. Bianchettin, A. Baraldi, S. de Gironcoli, S. Lizzit, L. Petaccia, E. Vesselli, G. Comelli, and R. Rosei, Phys. Rev. B 74, 045430 (2006).

[26] T. Nowitzki, A. F. Carlsson, O. Martyanov, M. Naschitzki, V. Zielasek, T. Risse, M. Schmal, H.-J. Freund, and M. Bäumer, J. Phys. Chem. C 111, 8566 (2007). 


\section{Supplementary Material}

\section{Experimental details}

Scanning tunneling microscopy measurements (STM) have been carried out in Graz, in an ultrahigh vacuum (UHV) custom-designed variable-temperature STM system (Oxford Instruments) with a base pressure below $10^{-10}$ mbar, equipped with the typical facilities for sample manipulation and cleaning and for physical vapor deposition [1]. For the present measurements, the STM was operated at room temperature in constant current mode using electrochemically prepared W tips, which have been cleaned in situ by electron bombardment. Detailed tunneling conditions are given at the respective images, with positive sample bias leading to empty electron state imaging of the sample. High-resolution $\mathrm{x}$-ray photoelectron spectroscopy (HR-XPS) experiments have been performed at beamline I311 in the Swedish synchrotron radiation laboratory MAX-Lab, Lund, Sweden [2]. The experimental end-station, which consists of two separate preparation and analysis chambers in a vertical mount, is equipped with standard surface science facilities for sample preparation, LEED optics, and a hemispherical SCIENTA-SES200 electron energy analyzer. The photoemission spectra were taken at photon energies of 140 and $950 \mathrm{eV}$ in order to excite photoelectrons from the $\mathrm{Al} 2 \mathrm{p}$ and $\mathrm{Ni} 2 \mathrm{p}$ core level regions, respectively. The total energy resolution in these experiments was 100-200 meV. All core level spectra were collected with the sample held at room temperature (300 K). The binding energy scale was calibrated with respect to the Fermi energy of the metal substrate, and all spectra were normalized to the secondary electron background at a few eV lower binding energy than the respective core level peak. A linear (Shirley) background was subtracted from the Al $2 p(\mathrm{Ni} 2 p)$ core level spectra prior to the peak decomposition analysis. The latter has been performed with the help of commercial least-squares minimization software FitXPS [3]. The core-level spectra have been decomposed into several components, whose individual shape consists of a Donjach-Šunjić profile [4] convoluted with a Gaussian distribution.

The $\mathrm{Ni}_{3} \mathrm{Al}(111)$ single crystal surface was cleaned by repeated cycles of $\mathrm{Ar}^{+}$-ion sputtering $(1 \mathrm{keV})$ at room temperature and subsequent annealing for 7 min at $850^{\circ} \mathrm{C}$ 
and $730^{\circ} \mathrm{C}$. The alumina film was prepared by exposing the clean $\mathrm{Ni}_{3} \mathrm{Al}(111)$ surface at an oxygen partial pressure of $3 \times 10^{-8}$ mbar for 30 min with the sample kept at $700^{\circ} \mathrm{C}$, followed by $5 \mathrm{~min}$ annealing in $\mathrm{UHV}$ at $750^{\circ} \mathrm{C}$. This procedure was repeated several times until a well-ordered oxide film was obtained, as monitored by lowenergy electron diffraction (LEED) and STM measurements. Pd and Ni metals were deposited from a commercial electron beam evaporator (Focus EFM3), with the evaporation rate calibrated by a quartz microbalance. Nanodot arrays of $\mathrm{Ni}$ clusters have been formed by physical vapour deposition (PVD) of $\mathrm{Ni}$ at $200^{\circ} \mathrm{C}$ onto the alumina template. The Ni deposition was preceded by a "seeding" procedure [5], where $2.07 \times 10^{13}$ atoms $/ \mathrm{cm}^{2} \mathrm{Pd}$ (which amount is equivalent to $\sim 3 \mathrm{Pd}$ atoms per alumina unit cell) was evaporated at room temperature, to promote the $\mathrm{Ni}$ metal cluster nucleation [6].

Fig. S1 shows STM images of Ni NP preparations for Ni surface coverages of $9.4 \times 10^{14}$ atoms $/ \mathrm{cm}^{2}$ (a) and $2.3 \times 10^{15}$ atoms $/ \mathrm{cm}^{2}$ (b) and after oxidation of the corresponding system to NiO NP, (c) and (d), respectively. Note that the aspect (height/diameter) ratios of the NP of $\sim 1 / 2$ are consistent with particles of approximately hemisperical shapes. The average volumes of the NP were evaluated from large scale STM images by numerical integration of pixel volume of a large number of particles.

\section{Computational details}

DFT calculations were performed using the QuantumEspresso package [10], employing a basis set of plane waves, the Perdew-Burke-Ernzerhof (PBE) exchangecorrelation (xc-) functional [11] and ultrasoft pseudopotentials [12]: 6, 3 and 10 electrons were explicitly described for Oxygen, Aluminium and Nickel, respectively; a pseudopotential for Aluminium with the explicit description of 11 electrons was also used in selected calculations. Values of $40 \mathrm{Ry}$ and $240 \mathrm{Ry}$ were chosen as the energy cutoff for the selection of the plane waves for the description of the wave function and the electronic density, respectively. The PDOS were evaluated by using Lowdin projections of the wave function onto atomic orbitals, and used for a qualitative analysis of the trends in the chemical shifts in the Janak approximation, 
see Fig. $4 \mathrm{~b}$ in the main paper. More refined calculations using a final-state excited pseudo-potential approach [13] were performed to evaluate accurately the XPS chemical shifts for $O 1$ s and Al $2 p$ states, see Fig. $4 a$ (bottom).

The hexagonal unit cell was modeled by taking portions of the $\mathrm{Ni}_{3} \mathrm{Al}$ (111) surface, using a first-neighbor distance of $2.535 \AA$, corresponding to the experimental lattice parameter of the bulk alloy. To reduce the computational efforts associated with the huge $\mathrm{AlOx} / \mathrm{Ni3Al}(111)$ unit cell (entailing around 1200 atoms for a minimal description [14]) to a manageable size, we considered simplified models. Two different cells were considered: a small one, which contains three $\mathrm{Ni}$ atoms and one $\mathrm{Al}$ atom per layer and a big one, obtained by taking a unit cell four times larger than the small one. The support was described by taking eight (for the small cell) or four (for the big cell) layers in fcc stacking, corresponding to the $L 1_{2}$ chemical ordering of the bulk alloy; the positions of the atoms of the metal support were kept frozen in their bulk positions in all calculations. The oxide slab was symmetrically adsorbed on both sides of the metal support in order to cancel spurious coulombic interactions among replicated images. When considering pristine alumina, the oxide slab was composed of four layers alternating $\mathrm{Al}$ and $\mathrm{O}$ atoms (by exposing $\mathrm{Al}$ atoms at the interface with the metal support and terminated by $O$ atoms) in both $A B C A$ and $A B B A$ stacking (as described in the main paper) with a total stoichiometry of $\mathrm{Al}_{5} \mathrm{O}_{6}$. Both the PDOS and and the XPS chemical shifts evaluated according via the pseudopotential approach came out very similar in the $A B C A$ and $A B B A$ stackings. When considering an oxidized oxide film, the alumina slab was thickened by restoring the $\mathrm{Al}_{2} \mathrm{O}_{3}$ composition of the inner four layers of the $A B C A$ stacking mode and adding a 2-layer termination with an $\mathrm{Al}_{3} \mathrm{O}_{3}$ stoichiometry in both $\mathrm{ABCAAC}$ and $\mathrm{ABCABC}$ stacking modes. In this case we found a quantitative difference between the two stackings, with a larger difference between $\mathrm{Al}_{\mathrm{s}}, \mathrm{Al}_{\mathrm{s}-1}$ and $\mathrm{Al}_{\mathrm{i}}$ peak positions in the ABCAAC stacking, which thus turned out to be a less accurate model of the oxidized alumina slab. In all cases, the positions of the atoms of the oxide were fully relaxed until the forces resulted smaller than $0.01 \mathrm{eV} / \AA$. Along the $z$ axis, a minimum empty space of $15 \AA$ was chosen to avoid interactions between replicated cells. The first Brillouin zone was k-sampled by employing a $(4,4,1)$ grid (for the small cell) or a $(2,2,1)$ grid (for the big cell) and the electronic levels were broadened with a Gaussian smearing of about $0.03 \mathrm{eV}$. In some selected calculations involving the big cell, the first 
Brillouin zone was k-sampled at $\Gamma$ the point only. Calculations at the $\Gamma$ point speed up the calculations still keeping a reasonable level of accuracy.

In the main text, the charge transfer from the substrate to the Ni NP has been discussed and the way it affects the system work function. In detail: the work function of the Ni3Al alloy of $4.8 \mathrm{eV}$ drops below $4 \mathrm{eV}$ after formation of the alumina film, thus becoming smaller than that of Ni NP (which is larger than $4 \mathrm{eV}$ already for a small $\mathrm{Ni13}$ cluster and rapidly increases to around 5-5.1 eV for bigger particles). This negative charging of the Ni NP causes the shift of the Al core levels to lower binding energies, as discussed in the main text. The further shift occurring at $45 \mathrm{~L}$ oxygen before appreciable oxidation of the Ni particles begins - can be interpreted as due to a thickening of the oxide layer. A structural model for this phenomenon is shown at the top of Fig. $4 \mathrm{~b}$, and consists of an $\mathrm{ABCABC}$ oxide slab obtained by adding an $\mathrm{Al}$ and a O layer in fcc stacking to the previous ABCA model.

\section{Model calculations of angular dependent Ni $2 p$ emission from a 2-D NP array}

To gain insight into the oxidation process of the NP from the evolution of the $\mathrm{Ni}^{0}$ and $\mathrm{Ni}^{2+}$ components after oxygen exposure (Figs. 3a,b), we have performed model calculations of the angular-dependent emission intensities from a two-dimensional (2-D) array of supported Ni NP. Two NP geometries have been considered: a 3-D rectangular box shape (Fig. S2a), which allows a relatively simple calculation of the $\mathrm{Ni}^{0}$ and $\mathrm{Ni}^{2+}$ intensities as a function of the oxide layer thickness, and a more realistic half-spherical shape (Fig. S2b), for which a more sophisticated analysis is necessary and which has been performed for the metallic and fully-oxidized NP only. Both NPs geometries exhibit the same volume and base area, the 3-D rectangular box has a square base with a side $\boldsymbol{b}$ and a height $\boldsymbol{d}_{2}$, while the half-sphere has a radius $\boldsymbol{R}$. The Ni particles are deposited onto a thin alumina film with a surface superperiodicity parameter $\boldsymbol{a}$ and a thickness $\boldsymbol{d}_{\mathbf{1}}$, which is supported on the $\mathrm{Ni}_{3} \mathrm{Al}(111)$ substrate. The NP occupy the regular "dot" sites of the AIOx film with a surface density $\boldsymbol{N}_{\boldsymbol{p}}$, thus forming a 2-D hexagonal array with a periodicity $\boldsymbol{a}$. In order to consider the NP as 
independent emitters of photoelectrons, the emission angle $\Theta$ should not exceed the limiting value $\Theta_{g}$, which is given by the formulas:

$$
\begin{array}{ll}
\tan \Theta_{g}=\frac{c}{a-b} & \text { - rectangular box } \\
\cos \Theta_{g}=\frac{R}{a-R} & \text { - half-sphere }
\end{array}
$$

For example, for half-sphere NP with radius $R=1.5 \mathrm{~nm}$ and $\mathrm{a}=4.1 \mathrm{~nm}$, is $\Theta_{\mathrm{g}} \approx 55^{\circ}$.

In order to eliminate the instrumental factors, the integrated Ni $2 p$ core level intensity is normalized to the signal of the underlying film-substrate (f-s) system, which is recorded prior to the particle deposition. The latter can be expressed as:

$$
I^{f-s}=\rho_{0} \lambda_{0} \exp \left(-\frac{d_{1}}{\lambda_{1} \cos \theta}\right)
$$

where $\rho$ and $\lambda$ denote the density and the electron inelastic mean free path (IMFP) in the different materials, and the indices 0 and 1 refer to the $\mathrm{Ni}_{3} \mathrm{Al}$ substrate and the AlOx film, respectively.

\section{3-D rectangular box particles}

The $\mathrm{Ni}^{0} 2 \mathrm{p}$ signal following the deposition of the Ni particles with a thickness $\mathrm{d}_{2}$ and an IMFP $\lambda_{2}$, is determined by the emitted intensity from the Ni atoms in the NP and the underlying $\mathrm{Ni}_{3} \mathrm{Al}$ substrate, the latter attenuated by the $\mathrm{AlOx}$ film and/or the $\mathrm{Ni}$ $\mathrm{NP}$, as given below:

$$
\begin{aligned}
I^{N i}= & \left\{\rho_{2} \lambda_{2}\left[1-\exp \left(-\frac{d_{2}}{\lambda_{2} \cos \theta}\right)\right]+\rho_{0} \lambda_{0} \exp \left(-\frac{d_{2}}{\lambda_{2} \cos \theta}\right) \exp \left(-\frac{d_{1}}{\lambda_{1} \cos \theta}\right)\right\} \\
& +(1-F) \rho_{0} \lambda_{0} \exp \left(-\frac{d_{1}}{\lambda_{1} \cos \theta}\right)
\end{aligned}
$$

where $F=N_{p} b^{2}$ is the fraction of the surface covered by the Ni particles.

Assuming next that in the course of the oxidation the Ni particles become covered by a layer of $\mathrm{NiO}$ with a thickness of $\mathrm{d}_{3}$, the overall $\mathrm{Ni}^{0} 2 \mathrm{p}$ intensity modifies to: 


$$
\begin{aligned}
I^{N i}=F \exp ( & \left.-\frac{d_{3}}{\lambda_{3} \cos \theta}\right)\left\{\rho_{2} \lambda_{2}\left[1-\exp \left(-\frac{d_{2}}{\lambda_{2} \cos \theta}\right)\right]\right. \\
& \left.+\rho_{0} \lambda_{0} \exp \left(-\frac{d_{2}}{\lambda_{2} \cos \theta}\right) \exp \left(-\frac{d_{1}}{\lambda_{1} \cos \theta}\right)\right\} \\
& +(1-F) \rho_{0} \lambda_{0} \exp \left(-\frac{d_{1}}{\lambda_{1} \cos \theta}\right)
\end{aligned}
$$

with the first two terms of Eq. (2) attenuated by the NiO overlayer, where $\lambda_{3}$ denotes the electron IMFP in NiO.

For the completely oxidized $\mathrm{NiO}$ particles $\left(\mathrm{d}_{2}=0\right)$ the $\mathrm{Ni}^{0} 2 \mathrm{p}$ intensity is determined only by the attenuated signal from the $\mathrm{Ni}_{3} \mathrm{Al}$ substrate:

$$
I^{N i}=F_{o x} \rho_{0} \lambda_{0} \exp \left(-\frac{d_{3}}{\lambda_{3} \cos \theta}\right) \exp \left(-\frac{d_{1}}{\lambda_{1} \cos \theta}\right)
$$

The corresponding $\mathrm{Ni}^{2+} 2 \mathrm{p}$ intensity due to the $\mathrm{NiO}$ layer is simply given by the expression:

$$
I^{N i O}=F_{o x} \rho_{3} \lambda_{3}\left[1-\exp \left(-\frac{d_{3}}{\lambda_{3} \cos \theta}\right)\right]
$$

\section{Half-spherical particles}

We first determine the intensity of photoelectrons emitted from a single particle in the direction $Y$ at a given angle $\Theta$, with respect to the surface normal (Z-axis) (Fig. S2). The intensity will depend on the coordinates $(x, y, z)$ of a point $P$ of a volume element inside the particle, the escape length $P Q$ of the electrons from the point $P$ to the particle's surface $Q$ and their inelastic mean free path $\lambda_{p}$ within the particle. The signal intensity from the particle can be obtained by the integration within the halfsphere:

$$
I_{p}=\rho_{p} \int_{-R}^{R} d x \int_{0}^{\sqrt{R^{2}-x^{2}}} d z \int_{-\sqrt{R^{2}-x^{2}-z^{2}}}^{\sqrt{R^{2}-x^{2}}} \exp \left(-\frac{P Q}{\lambda_{p}}\right) d y
$$


The escape length $P Q$ can be calculated by simple geometrical considerations to:

$$
P Q=\sqrt{R^{2}-x^{2}-(z \sin \theta-y \cos \theta)^{2}}-(z \cos \theta+y \sin \theta)
$$

For a single Ni particle the signal intensity is given by the expression:

$$
\begin{aligned}
I_{p} & =\rho_{2} \int_{-R}^{R} d x \int_{0}^{\sqrt{R^{2}-x^{2}}} d z \int_{-\sqrt{R^{2}-x^{2}-z^{2}}}^{\sqrt{R^{2}-x^{2}-z^{2}}} d y \\
& \exp \left(-\frac{\sqrt{R^{2}-x^{2}-(z \sin \theta-y \cos \theta)^{2}}-(z \cos \theta+y \sin \theta)}{\lambda_{2}}\right)
\end{aligned}
$$

The overall $\mathrm{Ni}^{0} 2 p$ signal $I^{N i}$ is given by the sum of the intensities of the particles $I_{p}^{N i}$ and the film-substrate $\mathrm{I}^{\mathrm{f}-\mathrm{s}}$, attenuated by the particles. To calculate this attenuation factor for a given emission angle $\Theta$, one has to integrate the path $P Q$ (note that here the point $P$ lies at the bottom of the particle, i.e. at $z=0$ ) over the half-sphere. The calculation of $P Q$ can be significantly simplified, by taking into account that the integration of $P Q$ in a sphere (or half-sphere) does not depend on the angle $\Theta . P Q$ can be at easiest calculated for $\Theta=0^{\circ}$ which yields:

$$
P Q=\sqrt{R^{2}-x^{2}-y^{2}}
$$

The attenuation factor due to a single particle can be obtained by performing the integration:

$$
A_{p}=\int_{-R}^{R} d x \int_{-\sqrt{R^{2}-x^{2}}}^{\sqrt{R^{2}-x^{2}}} \exp \left(-\frac{\sqrt{R^{2}-x^{2}-y^{2}}}{\lambda_{2}}\right) d y
$$

The overall intensity of the particles-film-substrate system can finally be written as:

$$
I^{N i}=F\left(I_{p}^{N i}+I^{f-s} A_{p}^{N i}\right)+(1-F) I^{f-s}
$$

where $F=N_{p} \pi R^{2}$ is the fraction of the surface covered by the Ni half-sphere NP.

After the oxidation of the Ni particles the $I^{N i}$ signal will be determined by the filmsubstrate signal $I^{f-s}$, attenuated by the corresponding $A_{p}^{\mathrm{NiO}}$ factor of the $\mathrm{NiO}$ particles: 


$$
I^{N i}=F_{o x} I^{f-s} A_{p}^{N i O}+\left(1-F_{o x}\right) I^{f-s}
$$

with $F_{o x}=N_{p} \pi R_{o x}^{2}$, where $\mathrm{R}_{\mathrm{ox}}$ denotes the radius of the $\mathrm{NiO}$ particles. Thus, if the AIOx film thickness does not change during the oxidation step, the $I^{N i} / I^{f-s}$ signal after the oxidation of the Ni particles should not depend on the emission angle. The experimentally observed small decrease of the normalized $\mathrm{Ni} 2 \mathrm{p}$ intensity at higher emission angles (Fig. 3(c), Fig. S4, bottom curve) can then be accounted in the analysis by an increase of the AIOx film thickness from initially $0.5 \mathrm{~nm}$ [7] to $0.75 \mathrm{~nm}$.

Finally, the intensity of the oxidic $\mathrm{Ni}^{2+} 2 \mathrm{p}$ component is straightforward to derive and is given by:

$$
I^{N i O}=F_{O x} I_{p}^{N i O}
$$

The IMFP's of the different materials have been calculated for a kinetic energy of 100 $\mathrm{eV}$ (as employed in the experiment) by using the NIST Electron Inelastic-Mean-FreePath Database [8] and are listed together with the corresponding densities in Table 1. The expressions (8) and (10) have been numerically integrated by using the Mathematica 8 software [9] for a given radius $\mathrm{R}$ of the particles and as a function of the emission angle $\Theta$.

In Fig. S4 the normalized $\mathrm{Ni}^{0}$ and $\mathrm{Ni}^{2+}$ intensities (symbols) of the Ni NP, measured prior and after oxidation with $180 \mathrm{~L}$ oxygen, have been plotted together with the theoretical intensities, calculated for a surface coverage of $80 \%$ of rectangular (solid lines) and spherical (dashed lines) $\mathrm{Ni}$ and NiO NP with diameters of $3.0 \mathrm{~nm}$ and 3.5 $\mathrm{nm}$, respectively, as a function of the emission angle. Both models reproduce well the angular dependence of the experimental $\mathrm{Ni}^{0}$ and $\mathrm{Ni}^{2+}$ intensities of the metallic and oxidized NP, with larger deviations occurring only at higher emission angles, where the NP cannot be considered as independent emitters of electrons anymore. This justifies the use of the simple rectangular box model of NP to calculate the evolution of the angular-dependent $\mathrm{Ni}^{0}$ and $\mathrm{Ni}^{2+}$ intensities for progressively increasing thickness of the oxide layer and compare it to the experimental data for different oxygen exposures - this is presented in Fig. 3(d). The good agreement between the calculated and experiment data suggests that the oxidation of the Ni NP proceeds in a layer-type fashion beginning at the NP's surface, so that an oxide shell - metal core 
NP geometry for intermediate oxygen doses can be obtained. For large oxygen doses the experimental $\mathrm{Ni}^{0}$ and $\mathrm{Ni}^{2+}$ intensities converge towards the model curves of the fully oxidized NP. 
Table 1: Density and electron inelastic mean free path (IMFP) values calculated for a kinetic energy of $100 \mathrm{eV}$ in different materials.

\begin{tabular}{|c|c|c|}
\hline Material & Density $\left(\mathbf{g ~ c m}^{-3}\right)$ & IMPF (nm) \\
\hline $\mathrm{Ni}_{3} \mathrm{Al}$ & 7.26 & 0.47 \\
\hline $\mathrm{Al}_{2} \mathrm{O}_{3}$ & 3.98 & 0.71 \\
\hline $\mathrm{Ni}$ & 8.91 & 0.41 \\
\hline $\mathrm{NiO}$ & 6.67 & 0.49 \\
\hline
\end{tabular}




\section{References:}

[1] S. Surnev, L. Vitali, M. G. Ramsey, F. P. Netzer, G. Kresse, and J. Hafner, Phys. Rev. B 61, 13945 (2000)

[2] R. Nyholm, J.N. Andersen, U. Johansson, B.N. Jensen, I. Lindau, Nucl. Instrum. Methods Phys. Res. Sect. A, 467-468, 320 (2001)

[3] FitXPS Version 2.12, D.L. Adams, Univ. of Aarhus, Denmark

[4] S. Donjach and M. Šunjić, J. Phys. C 3, 185 (1970)

[5] G. Hamm, C. Becker and C. R. Henry, Nanotechnology 17, 1943 (2006)

[6] M. Schmid, G.Kresse, A. Buchsbaum, E. Napetschning, S. Gritschneder, M. Reichling, and P. Varga, Phys. Rev. Lett. 99, 196104 (2007)

[7] U. Bardi and G. Rovida, Surf. Sci. 239 (1990) L511.

[8] C.J. Powell and A. Jablonski, NIST Electron Inelastic-Mean-Free-Path Database, Version 1.2, 2010.

[9] www.wolfram.com/mathematica/

[10] P. Giannozzi, S. Baroni, N. Bonini, M. Calandra, R. Car, C. Cavazzoni, D. Ceresoli, G. Chiarotti, M. Cococcioni, I. Dabo, A. Dal Corso, S. De Gironcoli, S. Fabris, G. Fratesi, R. Gebauer, U. Gerstmann, C. Gougoussis, A. Kokalj, M. Lazzeri, L. Martin-Samos, N. Marzari, F. Mauri, R. Mazzarello, S. Paolini, A. Pasquarello, L. Paulatto, C. Sbraccia, S. Scandolo, G. Sclauzero, A. P. Seitsonen, A. Smogunov, P. Umari, and R. M. Wentzcovitch, J. Phys.: Condens. Matter 21, 395502 (2009)

[11] J. P. Perdew, K. Burke, and M. Ernzerhof, Phys. Rev. Lett. 77, 3865 (1996)

[12] D. Vanderbilt, Phys. Rev. B 41, 7892 (1990)

[13] L. Bianchettin, A. Baraldi, S. De Gironcoli, S. Lizzit, L. Petaccia, Phys. Rev. B. 74, 045430 (2006)

[14] M. Schmid, G.Kresse, A. Buchsbaum, E. Napetschning, S. Gritschneder, M. Reichling, and P. Varga, Nanotemplate with holes: Ultrathin alumina on Ni3Al(111), Phys. Rev. Lett. 99, 196104 (2007) 


\section{Figure captions:}

Fig. S1: $\quad$ STM images $(200 \mathrm{~nm} \times 200 \mathrm{~nm}$; sample bias: $+3.0 \mathrm{~V}$; tunneling current: $0.1 \mathrm{nA}$ ) of Ni NP preparations for Ni coverages of: (a) $9.2 \times 10^{14} \mathrm{at} / \mathrm{cm}^{2}$ and (b) $2.3 \times 10^{15} \mathrm{at} / \mathrm{cm}^{2}$; STM images of the corresponding $\mathrm{NiO} \mathrm{NP}$ surfaces are shown in (c) $(150 \mathrm{~nm} \times 150 \mathrm{~nm}$; sample bias: $+3.1 \mathrm{~V}$; tunneling current: $0.1 \mathrm{nA})$ and (d) $(200 \mathrm{~nm} \times 200 \mathrm{~nm}$; sample bias: +3.2 $\mathrm{V}$; tunneling current: $0.05 \mathrm{nA}$ ).

Fig. S2: Schematic view of the NP geometries: (a) 3-D rectangular box and (b) half-sphere.

Fig. S3: The geometry to calculate the angular dependence of photoelectron intensity for half-sphere particles.

Fig. S4: Angular dependence of the integrated $\mathrm{Ni} 2 \mathrm{p}_{3 / 2}$ intensity normalized to the respective values of the bare $\mathrm{AlO}_{x} / \mathrm{Ni}_{3} \mathrm{Al}(111)$ surface. The squares denote the normalized $\mathrm{Ni}^{0}$ intensity before oxidation, whereas the circles and triangles correspond to the $\mathrm{Ni}^{\circ}$ and $\mathrm{Ni}^{2+}$ intensities, respectively, after oxidation with $180 \mathrm{~L} \mathrm{O}_{2}$ at $200^{\circ} \mathrm{C}$ (Ni surface coverage: $1.37 \times 10^{15}$ atoms $/ \mathrm{cm}^{2}$ ). The solid and dashed lines are the calculated angular dependence curves for particles with rectangular box and half spherical shapes, respectively. 

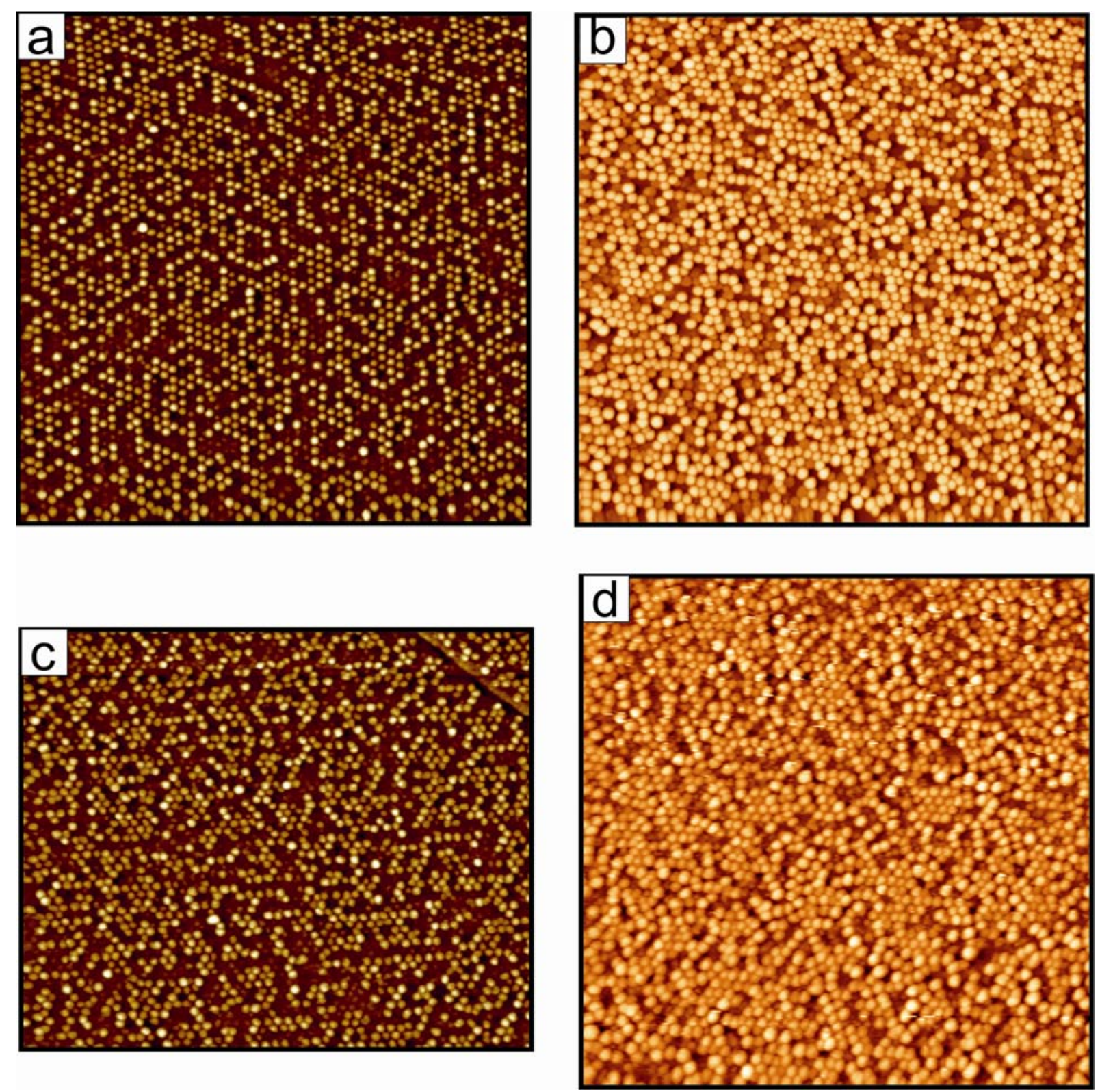

\section{Fig. S1}




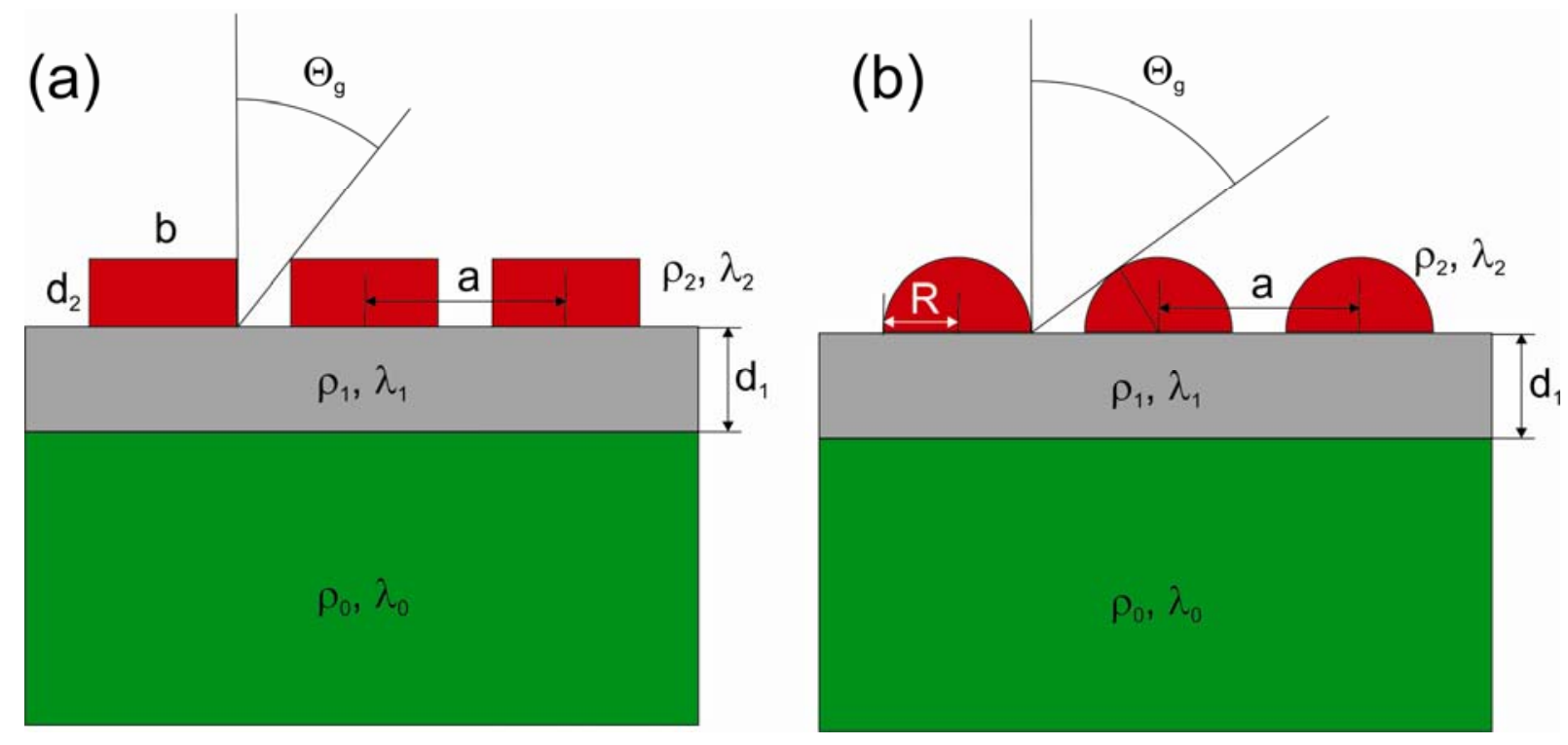

Fig. S2

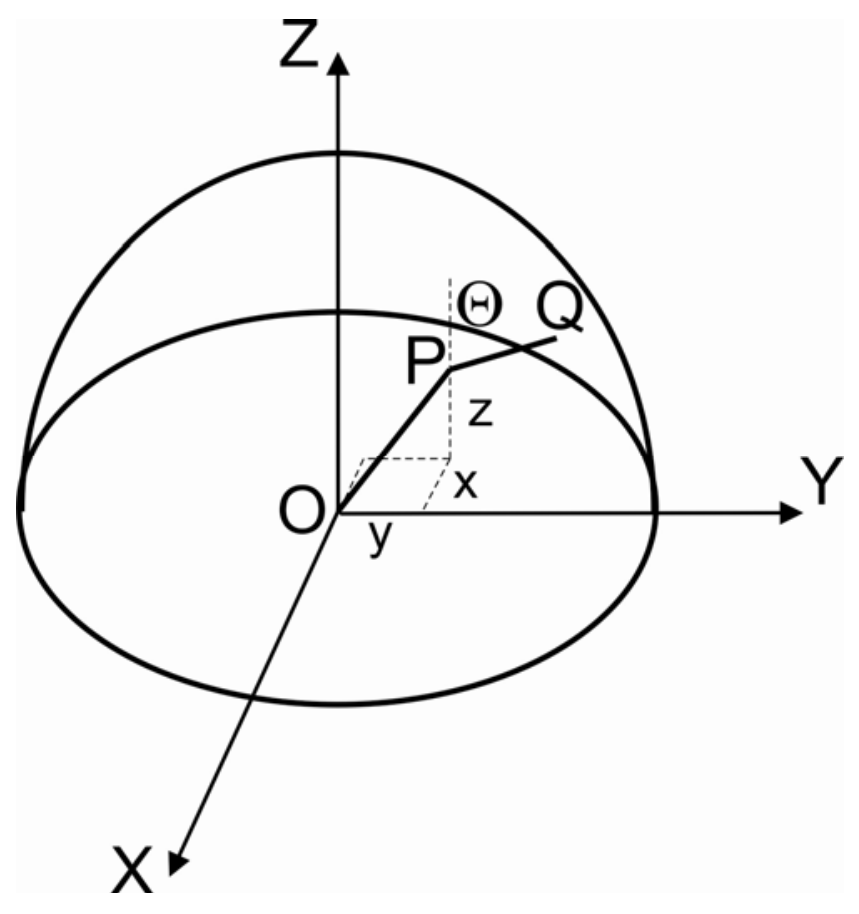

Fig. S3 


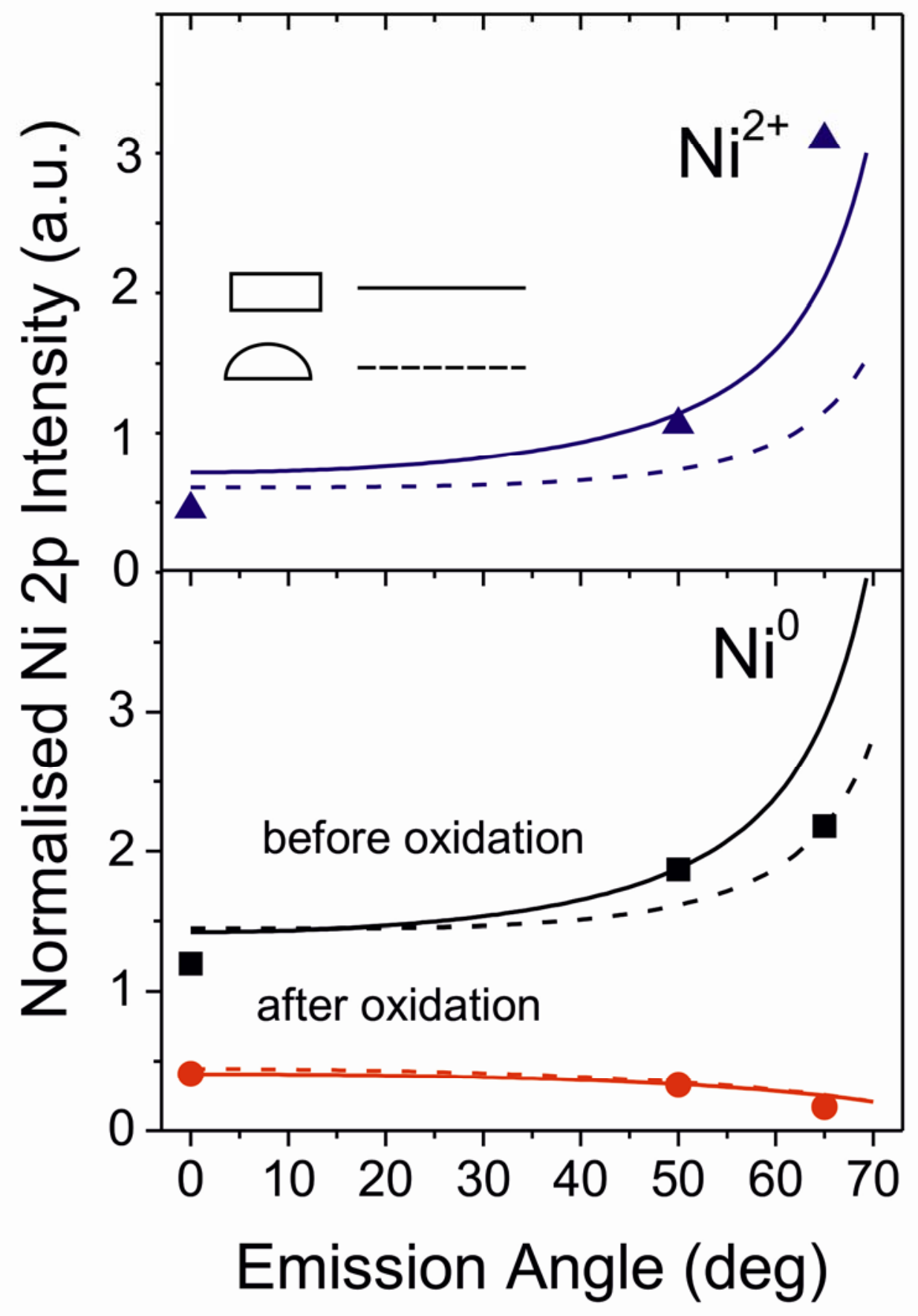

Fig. S4 